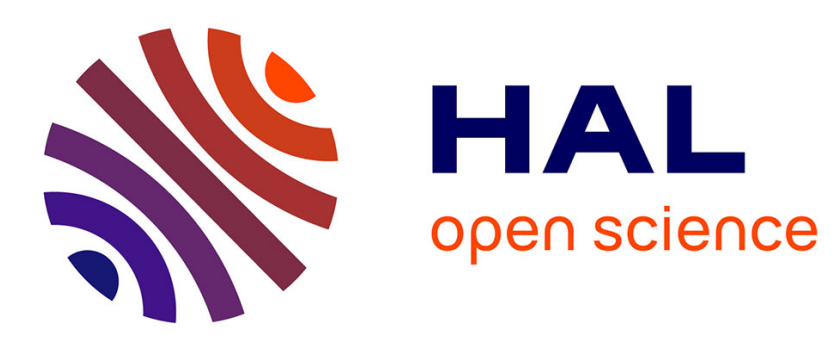

\title{
State and parameter estimation in 1-D hyperbolic PDEs based on an adjoint method
} van Tri Nguyen, Didier Georges, Gildas Besancon

\section{To cite this version:}

van Tri Nguyen, Didier Georges, Gildas Besancon. State and parameter estimation in 1-D hyperbolic PDEs based on an adjoint method. Automatica, 2016, 67, pp.185-191. 10.1016/j.automatica.2016.01.031 . hal-01236528

\section{HAL Id: hal-01236528 \\ https://hal.science/hal-01236528}

Submitted on 22 Dec 2016

HAL is a multi-disciplinary open access archive for the deposit and dissemination of scientific research documents, whether they are published or not. The documents may come from teaching and research institutions in France or abroad, or from public or private research centers.
L'archive ouverte pluridisciplinaire HAL, est destinée au dépôt et à la diffusion de documents scientifiques de niveau recherche, publiés ou non, émanant des établissements d'enseignement et de recherche français ou étrangers, des laboratoires publics ou privés. 


\title{
State and parameter estimation in 1-D hyperbolic PDEs based on an adjoint method *
}

\author{
Van Tri NGUYEN ${ }^{a}$, Didier GEORGES ${ }^{a}$, Gildas BESANCON ${ }^{a, b}$ \\ ${ }^{\mathrm{a}}$ Univ. Grenoble Alpes, GIPSA-Lab, F-38000 Grenoble, France \\ ${ }^{\mathrm{b}}$ Institut Universitaire de France
}

\begin{abstract}
An optimal estimation method for state and distributed parameters in 1-D hyperbolic system based on adjoint method is proposed in this paper. A general form of the partial differential equations governing the dynamics of system is first introduced. In this equation, the initial condition or state variable as well as some empirical parameters are supposed to be unknown and need to be estimated. The Lagrangian multiplier method is used to connect the dynamics of the system and the cost function defined as the least square error between the simulation values and the measurements. The adjoint state method is applied to the objective functional in order to get the adjoint system and the gradients with respect to parameters and initial state. The objective functional is minimized by Broyden-Fletcher-Goldfarb-Shanno (BFGS) method. Due to the non-linearity of both direct and adjoint system, the nonlinear explicit Lax-Wendroff scheme is used to solve them numerically. The presented optimal estimation approach is validated by two illustrative examples, the first one about state and parameter estimation in a traffic flow, and the second one in an overland flow system.
\end{abstract}

Key words: State estimation, Parameter estimation, Hyperbolic system, Adjoint method, Inverse problem, Saint-Venant model, Lighthill-Whitham-Richards model

\section{Introduction}

In nature and ordinary life, one can find a lot of physical laws described by hyperbolic partial differential equations of order one such as water flow, traffic flow, gas dynamics or electrical lines for instance. Researchers in control have investigated a large number of problems concerning this type of systems with different purposes for example to design an infinite-dimensional nonlinear predictive control for open-channel flow [8]; to model and control a dam-river system [14], [15]; to investigate the receding horizon boundary control applied on Lighthill-Whitham-Richards traffic flow model to avoid shock waves [19]; to optimally control traffic in highway

\footnotetext{
ऋ This work has been partially supported by the LabEx PERSYVAL-Lab (ANR-11-LABX-0025-01) and the MEPIERA project, Grenoble Institute of Technology

Email addresses:

van-tri.nguyen@gipsa-lab.grenoble-inp.fr (Van Tri NGUYEN),

didier.georges@gipsa-lab.grenoble-inp.fr (Didier GEORGES),

gildas .besancon@gipsa-lab.grenoble-inp.fr (Gildas BESANCON).
}

network using linear programming [12]; to apply the predictive control method on gas jet flames distribution [22]. One of the important problems arising in the simulation and control of hyperbolic systems is that uncertainty of the initial condition and empirical parameters can cause large errors and inconsistency between the output of control system and the real one. This motivated few studies on observer design, and even output feedback control, going back to works of Christofides et al $[3,4]$ for instance, with pole placement and Kalman designs , up to [9] more recently, with backstepping approaches (as also in [21]). In the present paper, the purpose is to develop a method based on optimal control theory to optimally estimate the initial condition and distributed parameters in such 1-D hyperbolic systems. Some authors studied constant parameter estimation in hyperbolic system such as Becker et al. who used the method of influence coefficient to estimate Manning roughness coefficient in an unsteady open-channel flow [2]; H. Longxi investigated a complex method to estimate the values of all roughness of a channel network [16]; Y. Ding et al. proposed an adjoint analysis method to find out roughness coefficient in shallow water [5]. In the case of distributed parameters, Y. Ding et al. also considered the same approach and applied it on the multi-reaches chan- 
nel flow network [6]; Richard et al. considered a numerical scheme used to solve parameter identification issue for 1-D hyperbolic system [7]; whereas Wenhuan investigated a quasi-Newton method to deal with the same problem [23]. In a former paper of Bagchi et al. [1], the parameter and system state of a discrete-time hyperbolic system with noisy boundary condition are estimated simultaneously based on the maximization of a likelihood function. More recently, we proposed an adjoint method to estimate the initial conditions in an overland flow described by a one dimensional Saint-Venant equation [18]. Notice that contemporaneously with it, Hasan et al. investigated a moving horizon technique to estimate state and constant parameter in a $2 \times 2$ linear hyperbolic system based on a distributed model for drilling application [10]. In that study, the model was discretized first and the adjoint method was applied to the resulting finite dimensional system. In the present paper, which can be considered as an extended version of [18] to general hyperbolic systems, the adjoint method is formulated and solved directly on nonlinear infinite dimensional models. More precisely, we deal with a parameter and state estimation approach in a one-dimensional nonlinear hyperbolic system, with a variable denoted by $u(x, t)$, and a flow denoted by $f(u(x, t), x)$, respectively depending on some initial condition denoted by $u_{0}^{i}(x)$, and some distributed parameters denoted by $\alpha_{i}(x)$, both supposed to be unknown, and thus needing to be estimated. The adjoint analysis is formulated with the original infinite dimensional system, to connect the sensitivity of variables needing to be estimated with the system model and the measurements. These points are also the main contributions of the present paper, and to the best of our knowledge, there are very few researches realized with such a spirit.

The rest of this paper is organized as follows: section 2 describes the dynamics of system and the formulation of optimal estimation problem. The adjoint method is applied to the optimization problem to get the adjoint system and gradient of estimated variables is presented in section 3. In section 4, two illustrative examples dealing with parameter and state estimation in traffic flow and overland flow system are presented. Some conclusions and perspectives are given at the end of the paper.

\section{Estimation problem statement}

\subsection{System dynamics}

Let us consider a general form of 1-D hyperbolic system of variable $u(x, t)$ and flow $f(u(x, t), x)$ a function of $u(x, t)$ and $x$. The spatial variable $x$ and time variable $t$ belong to the set $(x, t) \in[0, L] \times \mathbb{R}^{+}$, and the system reads:

$$
\left\{\begin{array}{l}
\frac{\partial u(x, t)}{\partial t}+\frac{\partial f(u(x, t), x)}{\partial x}=g(x, t) \\
u(x, 0)=u_{0}^{i}(x) \\
u(0, t)=u_{0}^{b}(t)
\end{array}\right.
$$

where the function $u_{0}^{b}(t)$ is a predefined boundary condition, the function $u_{0}^{i}(x)$ denotes the initial condition, and $g(x, t)$ is a known function. Notice that if it is clear enough in the sequel, notation $f$ and $u$ will be used instead of $f(u(x, t), x)$ and $u(x, t)$ in order to shorten the equation length. Let us assume, without any loss of generality, that the function $f(u, x)$ can be written in the following form for some vectors $\alpha=\left[\alpha_{1}(x) \ldots \alpha_{i}(x) \ldots \alpha_{K}(x)\right]^{T}$ and $\varphi=\left[\varphi_{i}(u) \ldots \varphi_{i}(u) \ldots \varphi_{K}(u)\right]^{T}$.

$$
f=\sum_{i=1}^{K} \alpha_{i}(x) \varphi_{i}(u)
$$

On this basis, let us consider the problem of estimating time and space evolution of $u$ when initial condition $u_{0}^{i}(x)$ is unknown, together with function parameter $\alpha(x)$. Once the initial state and parameter of system are successfully recovered, all transient state profiles of the system can be fully rebuilt by simulation.

\subsection{Optimal estimation problem}

For the estimation problem of parameter and state in the considered hyperbolic system, one can use two main approaches: empirical procedures or minimization approach based on optimal control theory. The first approach uses direct empirical formula with observation data to get the parameter value and is suitable only for simple parameter estimation. In the present work, due to the complexity and non linearity of estimation problem, we use the second approach to minimize the errors between simulations and some lumped observation values of variable $u(x, t)$. In other words, we minimize a cost function $J$ defined as follows:

$$
\begin{aligned}
J & =\frac{1}{2} \sum_{j=1}^{N} \int_{0}^{T}\left\{\int_{0}^{L} \delta_{A}\left(x-x_{j}\right) u d x-u_{j}^{\text {meas }}\left(x_{j}, t\right)\right\}^{2} d t \\
& +\frac{1}{2} \varepsilon_{1} \int_{0}^{L}\left\|u_{0}^{i}(x)-u_{0 F}^{i}(x)\right\|^{2} d x \\
& +\frac{1}{2} \varepsilon_{2} \sum_{i=1}^{K} \int_{0}^{L}\left\|\alpha_{i}(x)-\alpha_{i F}(x)\right\|^{2} d x
\end{aligned}
$$

where $T=$ optimization horizon (hours); $L=$ considered spacial length where the system takes place $(m) ; N$ $=$ number of observation values of $u(x, t) ; u_{j}^{\text {meas }}\left(x_{j}, t\right)=$ measured value of $u(x, t)$ at observation position $x_{j}$ with $x_{j} \in[0, L] ; \alpha_{i F}(x)=$ first guessed values of parameters; $u_{o F}^{i}=$ first guessed values of initial condition; $\varepsilon_{1}$ and $\varepsilon_{2}=$ weighting factor applied to first guessing term to calibrate the estimated value and the guessed one and adjust the scale of objective function. The term $\delta_{A}\left(x-x_{j}\right)$ is an approximation of Delta-Dirac function described by a Gaussian function with a very small variance $\sigma^{2}$ as $\delta_{A}\left(x-x_{j}\right)=e^{-\left(x-x_{j}\right)^{2} / \sigma^{2}}$. 


\section{Adjoint-based approach}

\subsection{Variational analysis}

From the previous analysis, the optimal values of state and parameter must minimize the cost function in equation (3) and satisfy also the system equation (1). This constraint and the continuity of the first partial derivative of both system dynamics and cost function lead to use the Lagrange multiplier with Lagrangian variable $\lambda(x, t)$, which allows to combine the system equation and cost function into only one new cost functional $\mathcal{L}(\lambda, \rho, \alpha)$, shortened to $\mathcal{L}$, as follows.

$$
\mathcal{L}=J+\underbrace{\int_{0}^{T} \int_{0}^{L} \lambda\left[\frac{\partial u}{\partial t}+\frac{\partial f}{\partial x}-g(x, t)\right] d x d t}_{\mathcal{A}}
$$

To deal with this optimization problem the common adjoint method is used in order to obtain the adjoint system and establish the gradient of cost functional with respect to the parameters and state needing to be estimated. These gradients describe the sensitivity of cost functions under the constraints of governing equation and variation of parameters. First of all, we take the first variation of the cost functional with respect to the system variable $u$, initial condition $u_{0}^{i}(x)$ and weighting factor $\alpha_{i}(x)$. The first variation $\delta J$ is given in equation (5).

$$
\begin{aligned}
\delta J & =\sum_{j=1}^{N} \int_{0}^{T} \int_{0}^{L} \delta_{A}\left(x-x_{j}\right)\left[\int_{0}^{L} \delta_{A}\left(x-x_{j}\right) u d x\right. \\
& \left.-u_{j}^{\text {meas }}\right] \delta u d x d t \\
& +\varepsilon_{2} \sum_{i=1}^{K} \int_{0}^{L}\left[\alpha_{i}(x)-\alpha_{i F}(x)\right] \delta \alpha_{i}(x) d x \\
& +\varepsilon_{1} \int_{0}^{L}\left[u_{0}^{i}(x)-u_{0 F}^{i}(x)\right] \delta u_{0}^{i}(x) d x
\end{aligned}
$$

Then using the integration by part technique the first variation of second term, called $\mathcal{A}$, of $\mathcal{L}$ can be obtained and presented in equation (6).

$$
\begin{aligned}
\mathcal{A} & =\left.\int_{0}^{L}[\lambda u]\right|_{0} ^{T} d x-\int_{0}^{T} \int_{0}^{L} u \frac{\partial \lambda}{\partial t} d x d t+\left.\int_{0}^{T}[\lambda f]\right|_{0} ^{L} d t \\
& -\int_{0}^{T} \int_{0}^{L} f \frac{\partial \lambda}{\partial x} d x d t-\int_{0}^{T} \int_{0}^{L} \lambda g d x d t
\end{aligned}
$$

The first variation of $\mathcal{A}$, calculated with respect to system variable $u(x, t)$, initial condition $u_{0}^{i}(x)$ and param- eters $\alpha_{i}(x)$, is developed as follows:

$$
\begin{aligned}
\delta \mathcal{A} & =\left.\int_{0}^{L} \lambda \delta u\right|_{0} ^{T} d x-\int_{0}^{T} \int_{0}^{L} \frac{\partial \lambda}{\partial t} \delta u d x d t+\left.\int_{0}^{T}\left[\lambda \frac{\partial f}{\partial u}\right] \delta u\right|_{0} ^{L} d t \\
& +\left.\sum_{i=1}^{K} \int_{0}^{T}\left[\lambda \frac{\partial f}{\partial \alpha_{i}(x)}\right] \delta \alpha_{i}(x)\right|_{0} ^{L} d t-\int_{0}^{L} \int_{0}^{L} \frac{\partial f}{\partial u} \frac{\partial \lambda}{\partial x} \delta u d x d t \\
& -\sum_{i=1}^{K} \int_{0}^{T} \int_{0}^{L} \frac{\partial f}{\partial \alpha_{i}(x)} \frac{\partial \lambda}{\partial x} \delta \alpha_{i}(x) d x d t
\end{aligned}
$$

All the terms multiplied by $\delta u$ in equations (5) and (7) is collected together and set to zero to cancel all the value of $\delta u$. This gives the first order of optimality condition or the adjoint system of adjoint variable $\lambda(x, t)$, described in equation (8):

$$
\begin{array}{r}
-\frac{\partial \lambda}{\partial t}-\frac{\partial f}{\partial u} \frac{\partial \lambda}{\partial x}+\sum_{j=1}^{N} \delta_{A}\left(x-x_{j}\right) \times\left[\int_{0}^{L} \delta_{A}\left(x-x_{j}\right) u d x\right. \\
\left.-u_{j}^{\text {meas }}\right]=0
\end{array}
$$

where $\frac{\partial f}{\partial u}=\sum_{i=1}^{K} \alpha_{i}(x) \frac{d \varphi_{i}(u)}{d u}$. The gradient of objective functional with respect to parameter $\alpha_{i}(x)$, formed by selecting all the terms related to $\delta \alpha_{i}(x)$ in previous equations of first variation, is not identical throughout the considered spacial domain $[0, L]$. At positions $x=0$ and $x=L$ the gradients of $\alpha_{i}(x)$ are presented in equations (9) and (10). At other positions, this gradient is in equation (11).

$$
\begin{gathered}
\frac{\partial \mathcal{L}}{\partial \alpha_{i}(0)}=-\int_{0}^{T} \lambda(0, t) \frac{\partial f}{\partial \alpha_{i}(0)} d t \\
\frac{\partial \mathcal{L}}{\partial \alpha_{i}(L)}=\int_{0}^{T} \lambda(L, t) \frac{\partial f}{\partial \alpha_{i}(L)} d t \\
\frac{\partial \mathcal{L}}{\partial \alpha_{i}(x)}=-\int_{0}^{T} \int_{0}^{L} \frac{\partial f}{\partial \alpha_{i}(x)} \frac{\partial \lambda}{\partial x} d x d t \\
+\varepsilon_{2} \int_{0}^{L}\left[\alpha_{i}(x)-\alpha_{i F}(x)\right] d x
\end{gathered}
$$

where $\frac{\partial f}{\partial \alpha_{i}(x)}=\varphi_{i}(u)$.

By using the same method, the gradient with respect to initial condition $u_{0}^{i}(x)$ is obtained and presented in equation (12).

$$
\frac{\partial \mathcal{L}}{\partial u_{0}^{i}(x)}=-\int_{0}^{L} \lambda(x, 0) d x+\varepsilon_{1} \int_{0}^{L}\left[u_{0}^{i}(x)-u_{0 F}^{i}(x)\right] d x
$$


The system dynamics and adjoint equations must be discretized in order to be solved numerically. The considered spacial domain $[0, L]$ is discretized into a set of smaller sections $\left[x_{j}, x_{j+1}\right]$. As a result the gradients of initial condition and parameters $\alpha_{i}(x)$ at position $x_{j}, \forall x_{j}=j \triangle x$ where $0<j \leq M-1$ with $\triangle x=L / M$, $M$ being the number of point, become:

$$
\begin{gathered}
\frac{\partial \mathcal{L}}{\partial u_{0}^{i}\left(x_{j}\right)}=-\lambda\left(x_{j}, 0\right)+\varepsilon_{1}\left[u_{0}^{i}\left(x_{j}\right)-u_{0 F}^{i}\left(x_{j}\right)\right] \\
\frac{\partial \mathcal{L}}{\partial \alpha_{i}\left(x_{j}\right)}=-\left.\int_{0}^{T} \frac{\partial f}{\partial \alpha_{i}(x)} \frac{\partial \lambda}{\partial x}\right|_{x=x_{j}} d t+\varepsilon_{2}\left[\alpha_{i}\left(x_{j}\right)-\alpha_{i F}\left(x_{j}\right)\right]
\end{gathered}
$$

According to the optimization condition, the gradient of $u_{0}^{i}(x)$ must be zero to minimize the cost function. We can obtain this condition, through optimization process, by setting all the gradient at each discretization point $x_{j}$ to zero.

After forming the adjoint system and gradients, all the remaining terms of equations (5) and (7) are gathered together and set to zero to satisfy the optimization conditions and to get the initial and boundary condition of adjoint variable $\lambda(x, t)$.

$$
\begin{gathered}
\int_{0}^{L} \lambda(x, T) \delta u(x, T) d t+\int_{0}^{T} \lambda(L, t) \frac{\partial f(u(L, t), L)}{\partial u(L, t)} \delta u(L, t) d t \\
-\int_{0}^{T} \lambda(0, t) \frac{\partial f(u(0, t), 0)}{\partial u(0, t)} \delta u(0, t) d t=0
\end{gathered}
$$

where $\frac{\partial f}{\partial u}(L, t)=\sum_{i=1}^{K} \alpha_{i}(L) \frac{\partial \varphi}{\partial u}(L, t)$. The boundary condition of system variable $u(0, t)$ is fixed beforehand, as a result its variation $\delta u(0, t)$ is zero for all values of $t$. The first and second terms of equation (15) also vanish also if we make $\lambda(x, T)$ equal to 0 and $\lambda(L, t)$ must satisfy equation (16).

$$
\lambda(L, t) \sum_{i=1}^{K} \alpha_{i}(L) \frac{\partial \varphi}{\partial u}(L, t)=0 \forall(x, t) \in[0, L] \times \mathbb{R}^{+}
$$

They are the necessary conditions to solve the adjoint system (8). We can notice that the boundary condition $\lambda(L, t)$ of adjoint system depends on the value of the partial derivative of function $\varphi(u(L, t))$ with respect to system variable at position $L$. If this derivative does not vanish, then, $\lambda(L, t)$ must be equal to zero. This makes the gradient of parameter $\alpha_{i}(L)$ in equation (10) equal to zero and we can not estimated the value of parameter $\alpha_{i}(L)$. We can also notice that the gradient of initial condition $u_{o}^{i}(L)$ in equation (13) becomes much smaller compared to other positions because we only have the correction term with calibration factor $\epsilon_{1}$. With small $\epsilon_{1}$ the estimation of $u_{o}^{i}(L)$ does not converge. However, an $\varepsilon_{1}$ large enough can reduce the biases on the estimation of $u_{o}^{i}\left(x_{i}\right), \forall x_{j}=j \triangle x$ where $0 \leq j \leq M-1$ with $\triangle x=L / M, M$ being the number of point. In two numerical examples presented later on in section 4 , the problem of null value of $\lambda(L, t)$ is met. A temporary solution is to consider that the values of $u_{o}^{i}(L)$ and $\alpha_{i}(L)$ are measured, and to only deal with the estimation distributed in the internal spatial section $\forall x_{j}=j \triangle x$ where $0 \leq j \leq M-1$ with $\triangle x=L / M, M$ being the number of point. A specific analysis will be carried on to investigate more deeply this incompleteness of adjoint method for estimating the value of variable at the end of the considered domain.

\subsection{Numerical implementation}

\subsubsection{Explicit Lax-Wendroff scheme for discretization}

The Lax-Wendroff scheme belongs to the class of conservative schemes and was presented in 1960 by P.D. Lax and B. Wendroff [11]. It is a numerical method for solving the systems of one dimension hyperbolic conservation laws with second order of accuracy in both space and time. It can be easily extended to the case of general 1-D hyperbolic system (1) with non homogeneous term $g(x, t)$. The formula of this scheme can be derived by a variety of approaches including Taylor series expansion in time of variable $u(x, t)$ :

$$
u(x, t+\triangle t)=u(x, t)+\triangle t \frac{\partial u}{\partial t}+\frac{\triangle t^{2}}{2} \frac{\partial^{2} u}{\partial t^{2}}+\mathcal{O}\left(\triangle t^{2}\right)
$$

The first and second partial derivative of $u(x, t)$ with respect to $t$ can be replaced by space derivatives of and others term of equation (1), which are assumed to exist with enough smoothness. The value of variable $u$ at time $t+\triangle t$ is as follows, neglecting the higher order terms $\mathcal{O}\left(\triangle t^{2}\right)$ in Taylor series expansion :

$$
\begin{aligned}
& u(x, t+\Delta t)=u(x, t)+\Delta t\left[-\frac{\partial f}{\partial x}+g\right]+\frac{\Delta t^{2}}{2} \\
& \times\left[-\frac{\partial \frac{\partial f}{\partial u}}{\partial x}\left[-\frac{\partial f}{\partial x}+g\right]-\frac{\partial f}{\partial u}\left[-\frac{\partial^{2} f}{\partial x^{2}}+\frac{\partial g}{\partial x}\right]+\frac{\partial g}{\partial t}\right]
\end{aligned}
$$

All the space and time derivatives in equation (18) are approximated by central differences. By denoting $u(x+$ $i \triangle x, t+n \triangle t)$ by $u(i, j)$ and $\frac{\partial f}{\partial u}=h$, the Lax-Wendroff scheme for discretizing equation (1) becomes:

$$
\begin{aligned}
& u(i, n+1)=u(i, n)+\triangle t\left[-\frac{f(i+1, n)-f(i-1, n)}{2 \triangle x}\right. \\
& +g(i, n)]+\frac{\triangle t^{2}}{2}\left\{-\frac{h(i+1, n)-h(i-1, n)}{2 \triangle x}\right. \\
& \times\left[-\frac{f(i+1, n)-f(i-1, n)}{2 \triangle x}+g(i, n)\right]-h(i, n) \\
& \times\left[-\frac{f(i+1, n)-2 f(i, n)+f(i-1, n)}{\triangle x^{2}}\right.
\end{aligned}
$$


$\left.\left.+\frac{g(i+1, n)-g(i-1, n)}{2 \triangle x}\right]+\frac{g(i, n+1)-g(i, n-1)}{2 \triangle t}\right\}$

The conventional truncation error analysis shows that this 3-point scheme is second-order accurate in space and time. Moreover, its stability and convergence is guaranteed by the Courant-Friedrichs-Lewy (CFL) condition. In our case of one-dimensional hyperbolic system, this CFL reads $\left|u_{\max }\right| \frac{\triangle t}{\triangle x} \leqslant C_{\max }$. The typical value of coefficient $C_{\max }$ for this explicit Lax-Wendroff scheme is 1 . It is worth noting that this scheme has also been chosen after comparison of its computational burdern with other possible approaches. In particular, an alternative implicit four-point Preissman scheme was considered (see [18]) but appeared to be more time consuming. For example, to numerically simulate the traffic flow of subsequent subsection 4.1.1 (with 10 space discretization steps and 300 time discretization steps), it takes $0.24 \mathrm{sec}-$ onds with Lax-Wendroff scheme, and 10.67 seconds with Preissmann scheme, using the same simulation parameters and same computation power (Intel(R) Core(TM)2 Duo CPU P8600 @2.40 GHz, 3Go RAM with Matlab R2011b 32 bit).

\subsubsection{Method for optimal estimation problem resolution}

The optimization problem is solved by using optimization tool called fmincon in Matlab. The gradient of cost functional with respect to the initial condition and parameters got from equations $(9),(10),(14)$ and (13) is provided to this function as well. In order to prevent the estimated variables for $u_{0}^{i}(x)$ and $\alpha_{i}(x)$ to become negative and be out of the limits describing the physical meaning, we imposed also a linear constraint. It is worth to notice that the fmincon tool has itself a finite difference method to calculate the gradient of estimated variable, which means that it is not obligatory to provide it via adjoint method in some simple estimation problem. In our two examples, due to the estimation size and the complexity of considered nonlinear hyperbolic models, the optimization problem converges with long estimation time (in the case of traffic flow) or does not converge (in the case of overland flow) without feeding gradients.

\section{Illustrative examples}

In order to validate the effectiveness of the proposed method, we present hereafter two numerical examples taken from two different application fields: traffic flow dynamics on the one hand and overland flow dynamics on the other hand. The validation procedure is developed using free-error approach which means that all the measurements or observed values of system variable $u(x, t)$ is got from the exact simulation of system with optimal initial state and parameters without noise adding. These measurements are used as input of optimization process.
The accuracy of estimation result depends on the number and position of observation values. In this work, the number of sensors and their positions are selected manually, and a full sensitivity analysis of distributed state and parameter estimation with respect to the observations is left for future works.

\subsection{State and lumped parameter estimation for traffic flow}

\subsubsection{Dynamics of traffic flow system}

The traffic flow here-considered is described by the well-known Lighthill-Whitham-Richards (LWR) model $[13,20]$ which was firstly introduced by Lighthill and Whitham in 1955 and complemented by Richards in 1956. This is a macroscopic traffic flow model in which the movement of an individual vehicle is neglected, and only a set of vehicles with its characteristics like density and average velocity, is considered to formulate the dynamics of traffic flow, as presented in equation (21).

$$
\frac{\partial \rho(x, t)}{\partial t}+\frac{\partial[\rho(x, t) v(x, t)]}{\partial x}=0
$$

where $x \in \mathbb{R} ; t \geq 0$ are space and time variables; $\rho(x, t)$ is the density of vehicles (vehicle $/ \mathrm{km}$ ) in $x$ at time $t$; $v(x, t)$ is the average velocity of vehicles in $x$ at time $t$ $(\mathrm{km} /$ hour $)$. We make an assumption that the considered highway is long enough to be divided into a set of small sections, each of them with its own parameters. Moreover, on each section the velocity only depends on density $\rho(x, t)$ through the simple function $(22)$ below. This means that when the highway is empty or $\rho=0$, the vehicles can get the maximal velocity $v=v_{\max }$. When the highway is full of vehicles $\rho=\rho_{\max }$, the vehicles can not move $v=0$.

$$
v(x, t)=v_{\max }\left[1-\frac{\rho(x, t)}{\rho_{\max }}\right]
$$

The parameters $v_{\max }$ and $\rho_{\max }$ are supposed to be constant. This assumption is reasonable in real high way whose characteristics do not vary a lot along its length. As a result, the flow function is $f(\rho, x)=v_{\max } \rho(x, t)-$ $v_{\max } \rho^{2}(x, t) / \rho_{\max }$. We consequently have the vectors of parameters $\alpha=\left[\begin{array}{ll}v_{\max } & v_{\max } / \rho_{\max }\end{array}\right]^{T}$ and $\varphi(\rho)=$ $\left[\rho(x, t)-\rho^{2}(x, t)\right]^{T}$ and for $f(\rho(x, t), x)$ :

$$
\begin{aligned}
& \frac{\partial f(\rho(x, t), x)}{\partial \alpha_{1}}=\rho(x, t) \\
& \frac{\partial f(\rho(x, t), x)}{\partial \alpha_{2}}=-\rho^{2}(x, t) \\
& \frac{\partial f(\rho(x, t), x)}{\partial \rho(x, t)}=\alpha_{1}-2 \alpha_{2} \rho(x, t)
\end{aligned}
$$

Let us insert the partial derivative of flow function $\frac{\partial f(\rho(x, t), x)}{\partial \rho}=\alpha_{1}-2 \alpha_{2} \rho(x, t)$ into equation (8). Then we can get the adjoint system corresponding to the direct traffic flow in equation $(21)$. The gradient of cost functional $\mathcal{L}$ with respect to initial condition of vehicle 
density is the same as equation (13). The sensitivity of constant parameters $\alpha_{1}$ and $\alpha_{2}$ is a little bit different with the previous general calculation and described as follows.

$$
\begin{aligned}
\frac{\partial \mathcal{L}}{\partial \alpha_{i}} & =\left.\int_{0}^{T}\left[\lambda \frac{\partial f}{\partial \alpha_{i}}\right]\right|_{0} ^{L} d t-\int_{0}^{T} \int_{0}^{L} \frac{\partial f}{\partial \alpha_{i}} \frac{\partial \lambda}{\partial x} d x d t \\
& +\varepsilon_{2} \int_{0}^{L}\left[\alpha_{i}-\alpha_{i F}\right] d x
\end{aligned}
$$

with the derivatives $\frac{\partial f}{\partial \alpha_{1}}=\rho(x, t)$ and $\frac{\partial f}{\partial \alpha_{2}}=-\rho^{2}(x, t)$

\subsubsection{Estimation result}

The estimation process for estimating the optimal values of initial condition of vehicles density and two parameters $\alpha_{1}$ and $\alpha_{2}$ is realized on a straight traffic road with characteristics presented in Table A.1. The real value of maximal vehicles velocity $\alpha_{1}(x)$ is $150(\mathrm{~km} / \mathrm{hour})$ and the parameter $\alpha_{2}$ is $0.5(\mathrm{~km} /$ hour $\times \mathrm{km} /$ vehicle $)$. The high way length $L=100(\mathrm{~km})$ is divided into 10 sections with spatial discretization step $\triangle x=10(\mathrm{~km})$. The real value of initial condition and parameters can be also found in table A.1. The boundary condition is a sum of four sinusoidal signals with different frequencies and phases. The initial value feed to the optimization tool is $\rho_{\text {init }}(x)=20$ (vehicle $\left./ \mathrm{km}\right) \quad \forall x \in[0 \ldots L]$, $\alpha_{1}=v_{\max }(x)=100$ (km/hour $), \alpha_{2}=v_{\max } / \rho_{\max }=$ $0.25(\mathrm{~km} /$ hour $\times \mathrm{km} /$ vehicle $)$. Both the first guessed value of initial condition and two parameters is set to zero. The stopping criterion of optimization tool fmincon is whenever the tolerance on the cost function value $J$ or tolerance on estimation variable is smaller than the termination tolerance values. We took just 1 observation value at the end of considered domain, from a simulation of direct system (21) with real value of initial state and parameters. One can observe in Fig. 1 the estimation results for $\rho(x, 0)$. It converges to the respective real values indeed with some small bias values at some positions in the considered spatial section $\forall x_{j}=j \triangle x$ where $0 \leq j \leq M-1$ with $\triangle x=L / M$, $M$ being the number of point. The estimated value of parameters $\alpha_{1}$ and $\alpha_{2}$ are 149.8782 and 0.4988 respectively. These estimation biases are possibly caused by the numerical errors on direct and adjoint system simulation and convergence limitation of BFGS method. The estimated value of maximal vehicles density can be easily found by simple division $\rho_{\max }=\alpha_{1} / \alpha_{2}$. At the end of optimal estimation process the found minimal value of cost function $J$ is 0.0074 slightly larger than zero, the theoretical value, due to estimation biases. The relative errors between the real values of estimation variables and estimated ones are $0.2330 \%, 0.3011 \%$, and $0.0812 \%$ respectively for $v_{\max }, v_{\max } / \rho_{\max }$ and $\rho(x, 0)$ which are pretty small. After some simulation realized to determine the sensitivity of estimation result with respect to the number and position of measurements,

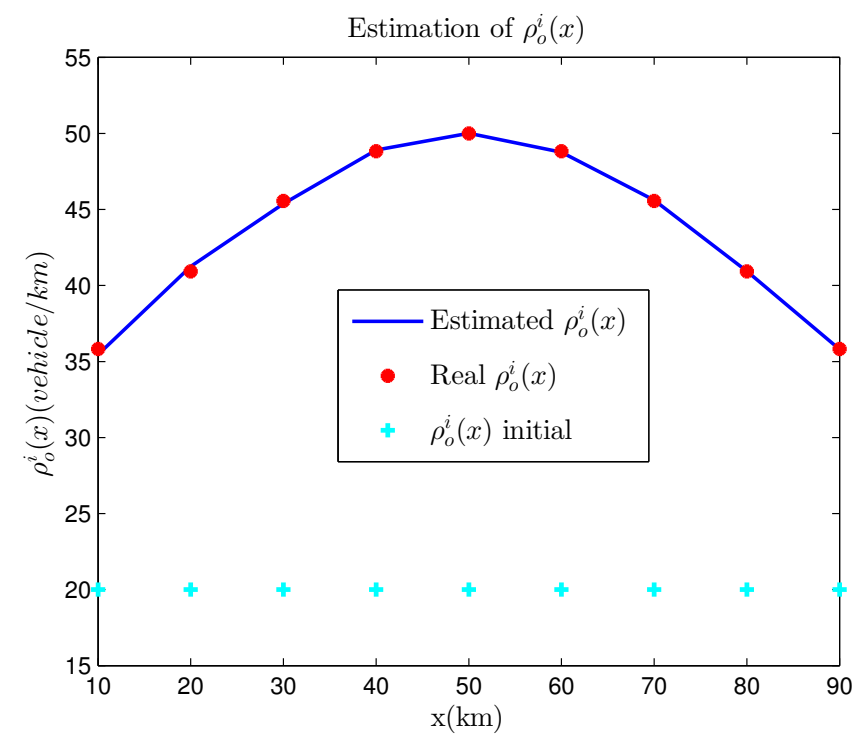

Fig. 1. Estimation of initial condition $\rho(x, 0)$

we can conclude that the more measurements we have, the more accurate result we obtain. But the accuracy improvement is not noticeable while being compared to the cost we pay for sensor number increment. Moreover, in this simulation, if the only one sensor is placed on the last position of considered domain, we obtain better optimization result than other positions.

\subsection{State and distributed parameter estimation for hy- draulic flow}

\subsubsection{Dynamics of over land flow system}

By definition, an overland flow is water, caused by excess rainfall or surface runoff, that flows over ground surface and towards some hydraulic channels. The dynamics of this type of water flow can be derived from the well-known Saint-Venant equations by some assumptions about small bottom slope, local and convective acceleration [18]. Finally the dynamics of overland flow is as follow:

$$
\left\{\begin{array}{l}
\frac{\partial h}{\partial t}+\frac{\partial f(h, x)}{\partial x}=r(x, t)-i(x, t) \\
h(x, 0)=h_{0}^{i}(x) \\
h(0, t)=h_{0}^{b}(t)
\end{array}\right.
$$

where $x=$ distance, $(m) ; t=$ time, $(s) ; h=$ water flow depth, $(m) ; f(h, x)=h^{5 / 3} S_{0}^{1 / 2} / n(x)=$ the flow per unit width $\left(\mathrm{m}^{2} / \mathrm{s}\right)$ with the spacial distributed Manning roughness coefficient $n(x) ; r=$ rainfall intensity, $(\mathrm{m} / \mathrm{s})$; $i=$ infiltration intensity, $(\mathrm{m} / \mathrm{s}) ; S_{0}=$ bed slope, $(\mathrm{m} / \mathrm{m})$. The infiltration $i(x, t)$ [17], caused by variable rainfall, can be described by the famous simple Green-Ampt model in equation (26):

$$
\begin{cases}i(t)=K_{i}\left(\frac{\Psi \eta(1-\theta)}{I(t)}+1\right) & \text { if } t>t_{p} \\ i(t)=r & \text { if } t \leq t_{p}\end{cases}
$$


where $K_{i}=$ effective hydraulic conductivity of the soil of flow, $\left(\frac{\mathrm{cm}}{\mathrm{s}}\right) ; \Psi=$ soil suction at wetting front, $(\mathrm{cm})$; $\eta=$ soil porosity, $(\%) ; \theta=$ relative initial soil moisture, (unitless); $I(t)=$ the cumulative infiltration, the accumulated depth of water infiltrating during the time period $t,(\mathrm{~cm}) ; t_{p}=$ ponding time, $(s)$ calculated by $t_{p}=\frac{\Psi K_{i} \eta(1-\theta)}{r\left(r-K_{i}\right)}$. By comparing this to the general form of flow in equation (2), we have the vector of only one parameter $\alpha(x)=S_{0}^{1 / 2} / n(x)$ and the corresponding function of system variable $\varphi(h)=h^{5 / 3}$. By inserting the derivative $\frac{\partial f(u, x)}{\partial h}=\frac{5}{3} \alpha(x) h^{2 / 3}$ into equation (8) we can obtain the adjoint system of overland flow. Replacing the derivative $\frac{\partial f(h, x)}{\partial \alpha(x)}=h^{5 / 3}$ into equations (9), (10) and (11), we can get the gradient of cost functional $\mathcal{L}$ with respect to this parameter.

\subsubsection{Estimation result}

This example is assumed to be taking place on some hills with characteristics described in Table. A.2. We need to estimate the initial condition of flow height $h(x, 0) \forall x \in$ $[0 \ldots L]$ whose real value is depicted in Fig. 2 and distributed parameter $\alpha$ whose real value $\alpha(x) \forall x \in[0 \ldots L]$, found via equation $\alpha(x)=S_{0}^{1 / 2} / n(x)$ with chosen values of $n(x)$, is in Fig. 3 . With the same estimation method as before, 2 observation values are taken out of 10 discretized sections ( 1 at the begin and 1 at the end) of considered domain and used as inputs for optimization process. The initial values of estimated state and parameter are $0.35(m) \forall x \in[0 \ldots L]$ and $7 \forall x \in[0 \ldots L]$ for, respectively, $h(x, 0)$ and $\alpha(x)$. After 192 iterations, the estimation of $h(x, 0)$ indeed converges to the desired value in Fig. 2 and parameter $\alpha(x)$ also converges in Fig. 3. The final value of cost function $J$ is $1.1478 \times 10^{-18}$ which is very small. Taking a look at the average relative errors of estimated values of $h_{o}^{i}(x)$ and $\alpha(x)$, respectively equal to $3.4240 \times 10^{-8 \%} \%$ and $2.4165 \times 10^{-8 \%}$, we can see that the estimation accuracy is pretty good. The estimated Manning coefficient $n(x)$, obtained via optimized parameter $\alpha(x)$, has also a small relative error $\left(3.4240 \times 10^{-8 \%}\right)$.

\section{Conclusions}

A general approach for parameter and state estimation in 1-D hyperbolic system based on optimal control theory and adjoint method has been developed and validated in this paper. First of all, the system dynamics and optimal estimation problem from lumped observation values have been formulated. Then the adjoint based method has been introduced to get the adjoint equation and gradients of objective functional, obtained by Lagrange multiplier method. From these gradients, the BFGS method has been used to find the optimal

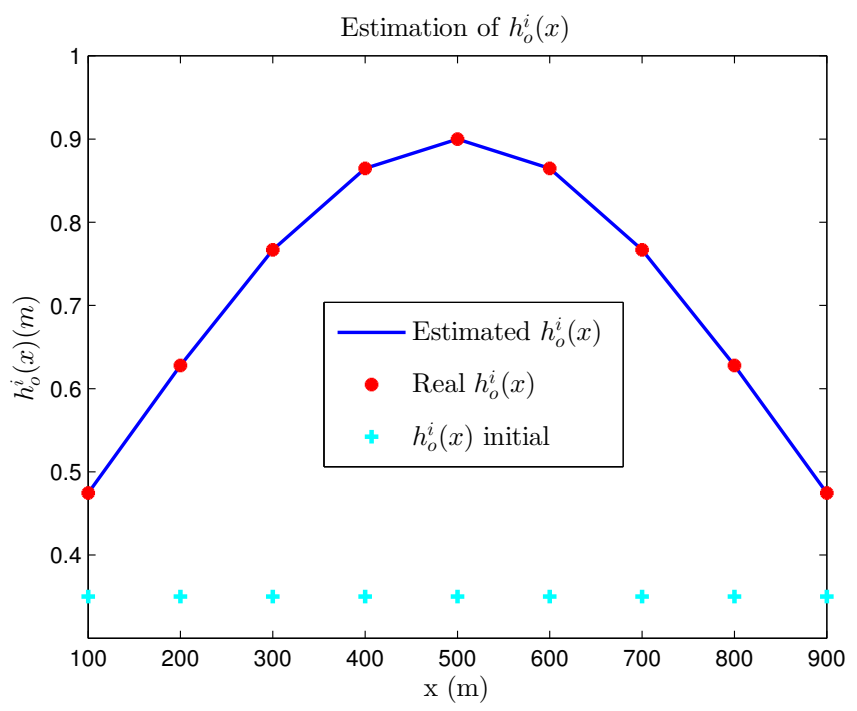

Fig. 2. Estimation of $h_{0}^{i}(x)$

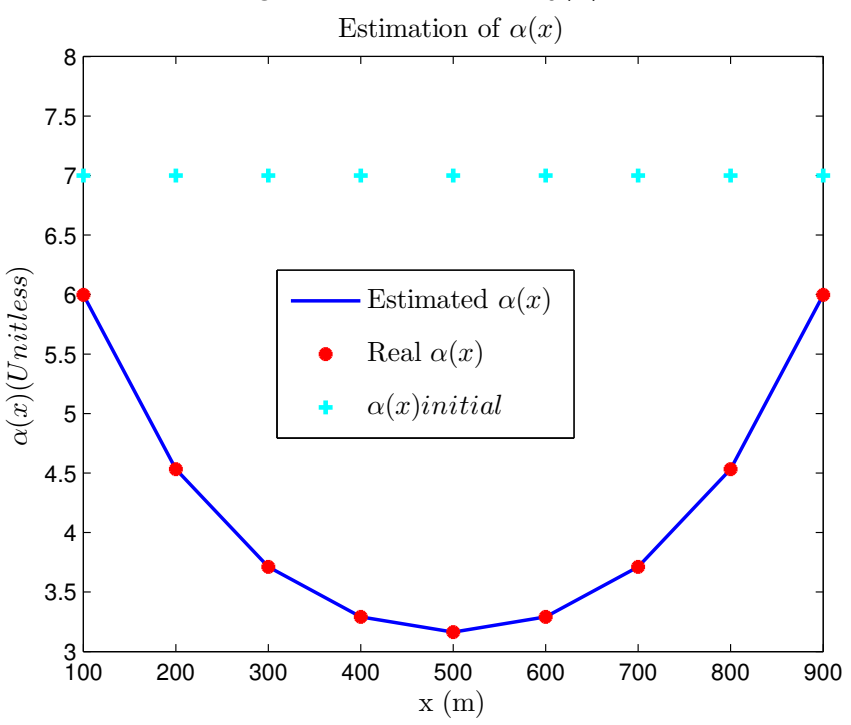

Fig. 3. Estimation of $\alpha(x)$

value of all parameter and state of variables which minimize the objective functional. The partial differential equations of both direct and adjoint system have been discretized and solved numerically by means of implicit Lax-Wendroff scheme. Finally, two numerical examples of hyperbolic system, overland flow modeled by SaintVenant equation and traffic flow modeled by LWR equation, have been provided to illustrate the effectiveness of the proposed approach. Because of its generality, this method can be applied to the state and parameter identification in any 1-D hyperbolic system. The observation data in this work are obtained from the simulation of system with optimal parameters and it my not represent the complexity and uncertainty of natural problem. As a result, a validation with real data or laboratory experiments is in progress to evaluate the applicability of the presented method to real problem. Moreover, more gen- 
eral problems such as output-feedback stabilization or optimal control with output constraints and time varying parameters will be part of our future developments.

\section{References}

[1] A. Bagchi and P. ten Brummelhuis. Simultaneous ML estimation of state and parameters for hyperbolic systems with noisy boundary conditions. In 29th IEEE Conference on Decision and Control, pages 222-224 vol.1, Honolulu, HI, 1990. IEEE.

[2] Leonard Becker and William W-G. Yeh. Identification of parameters in unsteady open channel flows. Water Resources Research, 8(4):956-965, August 1972.

[3] Panagiotis D. Christofides and Prodromos Daoutidis. Feedback control of hyperbolic PDE systems. AIChE Journal, 42(11):3063-3086, November 1996.

[4] Panagiotis D Christofides and Prodromos Daoutidis. Distributed output feedback control of two-time-scale hyperbolic PDE systems. International Journal of Applied Mathematics and Computer Science, 8:713-732, 1998.

[5] Yan Ding, Yafei Jia, and Sam S Y Wang. Identification of Manning's Roughness Coefficients in Shallow Water Flows. Journal of Hydraulic Engineering, 130(6):501-510, June 2004.

[6] Yan Ding and Sam S. Y. Wang. Identification of Manning's roughness coefficients in channel network using adjoint analysis. International Journal of Computational Fluid Dynamics, 19(1):3-13, January 2005.

[7] R.E. Ewing and Tao Lin. A direct method for parameter estimation in a hyperbolic partial differential equation. In 27th IEEE Conference on Decision and Control, pages 16621667, Austin, TX, 1988. IEEE.

[8] Didier Georges. Infinite-dimensional nonlinear predictive control design for open-channel hydraulic systems. Networks and Heterogeneous Media, 4(2):267-285, June 2009.

[9] Agus Hasan. Adaptive Boundary Control and Observer of Linear Hyperbolic Systems With Application to Managed Pressure Drilling. In ASME 2014 Dynamic Systems and Control Conference, San Antonio, TX, October 2014. ASME.

[10] Agus Hasan and Lars Imsland. Moving horizon estimation in managed pressure drilling using distributed models. In 2014 IEEE Conference on Control Applications (CCA), pages 605610, Juan Les Pins, Antibes, October 2014. IEEE.

[11] Peter Lax and Burton Wendroff. Systems of conservation laws. Communications on Pure and Applied Mathematics, 13(2):217-237, 1960.

[12] Yanning Li, Edward Canepa, and Christian Claudel. Optimal traffic control in highway transportation networks using linear programming. In 2014 European Control Conference (ECC), pages 2880-2887, Strasbourg, June 2014.

[13] Michael J Lighthill and Gerald Beresford Whitham. On kinematic waves. ii. a theory of traffic flow on long crowded roads. Proceedings of the Royal Society of London. Series A. Mathematical and Physical Sciences, 229(1178):317-345, 1955.

[14] X Litrico and D Georges. Robust continuous-time and discrete-time flow control of a dam-river system. (I) Modelling. Applied Mathematical Modelling, 23(11):809-827, November 1999.
[15] Xavier Litrico and Didier Georges. Robust continuoustime and discrete-time flow control of a dam-river system. (II) Controller design. Applied Mathematical Modelling, 23(11):829-846, November 1999.

[16] Han Longxi. Parameter estimation in channel network flow simulation. Water Science and Engineering, 1(1):10-17, 2008.

[17] Larry W Mays. Water resources engineering. John Wiley \& Sons, 2010.

[18] Van Tri Nguyen, Didier Georges, and Gildas Besancon. Optimal state estimation in an overland flow model using the adjoint method. In 2014 IEEE Conference on Control Applications (CCA), pages 2034-2039, Juan Les Pins, Antibes, October 2014. IEEE.

[19] Thang V. Pham, Didier Georges, and Gildas Besançon. Receding horizon boundary control of nonlinear conservation laws with shock avoidance. Automatica, 48(9):2244-2251, September 2012 .

[20] P.I Richards. Shock waves on the highway. Operations Research, 4(1):42-51, 1956.

[21] Rafael Vazquez, Miroslav Krstic, and Jean-Michel Coron. Backstepping boundary stabilization and state estimation of a $2 \times 2$ linear hyperbolic system. In IEEE Conference on Decision and Control and European Control Conference, volume 1, pages 4937-4942. IEEE, December 2011.

[22] Xubin Sun and Hong Wang. Dynamic System Control of Gas Jet Flames Distribution by Predictive Control Method. In 2005 IEEE International Symposium on, Mediterrean Conference on Control and Automation Intelligent Control, 2005., pages 1458-1463, Limassol, 2005. IEEE.

[23] Wenhuan Yu. A Quasi-Newton Method for Estimating the Parameter in a Nonlinear Hyperbolic System. Journal of Mathematical Analysis and Applications, 231(2):397-424, March 1999.

\section{A Tables of simulations parameters}

\begin{tabular}{|c|c|c|}
\hline Parameter & Value & Unit \\
\hline \hline Highway length $L$ & 100 & $\mathrm{~km}$ \\
\hline Observation horizon $T$ & 3 & hour \\
\hline Space step $\triangle x$ & 10 & $\mathrm{~km}$ \\
\hline Time step $\triangle t$ & 0.01 & hour \\
\hline Calibration coefficients $\epsilon_{1}=\epsilon_{2}=\epsilon_{3}$ & $1 \times 10^{-7}$ & Unitless \\
\hline Observation number $N$ & 1 & Unitless \\
\hline
\end{tabular}

\section{Table A.1}

Parameters used in traffic flow estimation example

\begin{tabular}{|c|c|c|}
\hline Parameters & Value & Unit \\
\hline \hline Length of plan & 1000 & $\mathrm{~m}$ \\
\hline Simulation time & 1000 & second \\
\hline Space step $\triangle x$ & 100 & $\mathrm{~m}$ \\
\hline Time step $\triangle t$ & 1 & second \\
\hline Bed slope $S_{0}$ & 0.001 & $\frac{m}{m}$ \\
\hline$K_{i}$ & 0.145 & $\frac{c m}{s}$ \\
\hline$\Psi$ & 21.85 & $\mathrm{~cm}$ \\
\hline$\eta$ & 0.434 & $\%$ \\
\hline$\theta$ & 0.463 & Unitless \\
\hline Calibration coefficient $\epsilon_{1}=\epsilon_{2}$ & $10^{-7}$ & Unitless \\
\hline Observation number $N$ & 2 & Unitless \\
\hline
\end{tabular}

Table A.2

Parameters used in overland flow estimation example 